\title{
Anisotropic Scattering Characteristics of a Radially Multilayered Gyrotropic Sphere
}

\author{
Lei Cao, Yongpin Chen, and Kai Kang \\ School of Electronic Engineering, University of Electronic Science and Technology of China, Chengdu 611731, China \\ Correspondence should be addressed to Kai Kang; kangkai@uestc.edu.cn
}

Received 24 April 2017; Accepted 24 July 2017; Published 28 September 2017

Academic Editor: Safieddin Safavi-Naeini

Copyright (C) 2017 Lei Cao et al. This is an open access article distributed under the Creative Commons Attribution License, which permits unrestricted use, distribution, and reproduction in any medium, provided the original work is properly cited.

\begin{abstract}
We present a new closed-form solution to the scattering of a monochromatic plane wave by a radially multilayered gyrotropic sphere using the $T$-matrix method. This approach can be utilized to investigate the interactions of a plane wave and a gyrotropic spherical scatterer of multiple layers with each layer characterized by both permittivity and permeability tensors. Based on the completeness and noncoplanar properties of vector spherical wave functions (VSWFs), analytical expressions of the electromagnetic fields in each gyrotropic layer are first derived. The boundary conditions are then applied on each discontinuous interface to obtain the scattering coefficients. Validations are made by first comparing the radar cross section (RCS) values of a 2-layered gyrotropic sphere with that computed from the full-wave finite element method (FEM) simulation and then reducing the general case to uniaxial case to compare the RCS values with the published results computed by Fourier transform combined with VSWFs method; in both cases good agreements are observed. Several specific cases are fully explored to investigate how the RCS are influenced by the parameters of the multilayered spherical structure. The results show that when both electric and magnetic gyrotropy tensors are considered, the RCS of the multilayered spherical scatterer can be suppressed or enhanced, depending on proper configurations of the material parameters.
\end{abstract}

\section{Introduction}

With the development of material science and fabrication techniques, the electromagnetic (EM) peculiarities of anisotropic media have attracted continued interests of both physics and engineering communities [1-7]. The effects of anisotropy or gyrotropy of the media must be taken into account in many practical applications, where a number of new devices with unique characteristics are designed by applying these effects $[8,9]$. For studying the interactions of EM waves and gyrotropic media, the most important and challenging task is to solve the vector wave equation characterizing EM fields in gyrotropic media. In the last several decades, a few analytical approaches have been developed based on the Cartesian coordinate expression of permittivity and permeability tensors, such as the perturbation expansion method $[10,11]$, the method of expanding EM fields in gyrotropic media using a complete set of VSWFs [12], the method of adopting VSWFs combined with Fourier transform [13-15], dyadic Green's functions method [16-18], and the T-matrix method [19-23]. To study the spherical anisotropy, Qiu et al. have investigated the characteristics of radial anisotropic uniaxial and gyrotropic sphere, where the permittivity and permeability tensors are expressed in spherical coordinate [24-30]. Due to the completeness and noncoplanar properties of VSWFs, any three-dimensional vector function satisfying the vector Helmholtz equation can be expanded as a linear combinations of VSWFs [31]. In [20], Lin and Chui have developed a complete theory to solve Maxwell equations for gyromagnetic particles. This method has a good solution accuracy since it does not involve lengthy evaluations of complicated integrals. Li and Ong applied this method to study the EM scattering characteristics of a gyroelectric sphere in [21] and then generalized the scattering problem by considering both permittivity and permeability tensors in [22]. In [23], the homogeneous gyroelectric sphere considered in $[20,21]$ was extended to a multilayered gyroelectric sphere.

In this paper, the method developed in [23] is further extended to the most general case: the gyrotropic sphere 


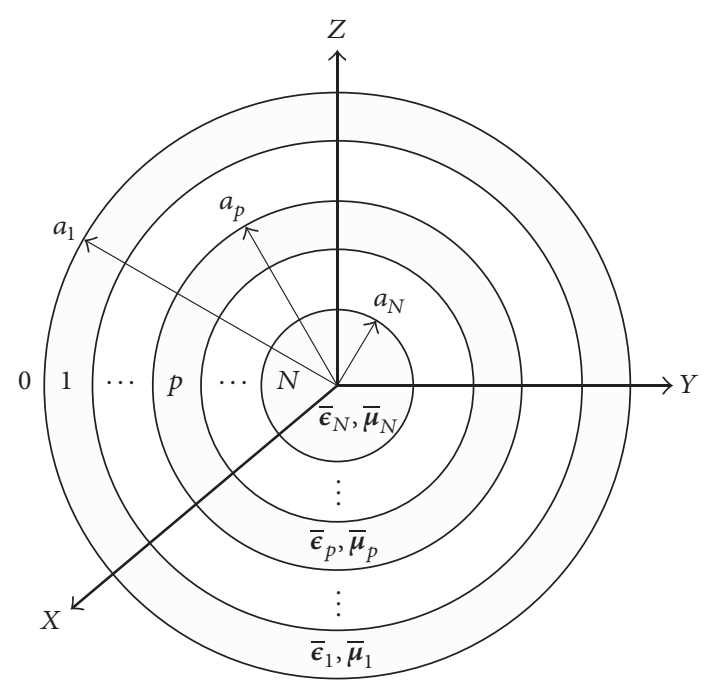

FIGURE 1: Geometry of a radially multilayered gyrotropic sphere.

under consideration consists of multiple anisotropic layers, each of which is characterized by both permittivity and permeability tensors. A new T-matrix formulation is proposed for the spherical multilayered scatterer. It is shown from numerical results that the proposed computation procedure is robust and accurate for a wide range of material parameters. In this method, the EM fields in each layer are first expanded as an infinite summation of VSWFs based on the Mie scattering theory $[20,32]$. The expansion coefficients in each layer and the scattering coefficients are then determined recursively by matching the boundary conditions at all interfaces. To validate the derived formulation, we compare two specific RCS results with those obtained by the FEM simulation and the Fourier transform combined with VSWFs method [33] for gyrotropic and uniaxial cases, respectively. Excellent agreements can be observed. The formulation developed in this paper can be used as a reference for other analytical approaches or numerical methods relying on discretizations. Finally, we calculate the RCS for several specific cases to provide some physical insights and possibilities of optimized designs for suppressing or enhancing RCS based on multilayered gyrotropic structures.

\section{Basic Formulations}

Throughout the paper, a time dependence $e^{-i \omega t}$ is assumed but always suppressed. The model of a radially multilayered gyrotropic sphere is depicted in Figure 1, where the radii of the spherical layers are denoted by $a_{1}, a_{2}, \ldots, a_{N}$. Region0 is the free space characterized by scalar permittivity $\left(\epsilon_{0}\right)$ and permeability $\left(\mu_{0}\right)$. Region- $p$ is homogeneous and characterized by both permittivity and permeability tensors, which are explicitly expressed in Cartesian coordinate as

$$
\overline{\boldsymbol{\epsilon}}_{p}=\epsilon_{p s}\left[\begin{array}{ccc}
\epsilon_{p r} & -i \epsilon_{p \kappa} & 0 \\
i \epsilon_{p \kappa} & \epsilon_{p r} & 0 \\
0 & 0 & 1
\end{array}\right]
$$

$$
\overline{\boldsymbol{\mu}}_{p}=\mu_{p s}\left[\begin{array}{ccc}
\mu_{p r} & -i \mu_{p \kappa} & 0 \\
i \mu_{p \kappa} & \mu_{p r} & 0 \\
0 & 0 & 1
\end{array}\right]
$$

where $p=1,2, \ldots, N$.

2.1. EM Fields in Multilayered Gyrotropic Sphere. In general, the EM fields in Region- $p$ of a multilayered gyrotropic sphere can be expressed as $[22,23]$

$$
\begin{aligned}
\mathbf{H}_{p} & =-\sum_{n, m} \bar{E}_{m n} \sum_{J} \sum_{l} \alpha_{p l}^{(J)} \frac{k_{p l}}{\omega \mu_{p s}}\left[\bar{d}_{m n, l}^{(p)} \mathbf{M}_{m n}^{(J)}\left(k_{p l}, \mathbf{r}\right)\right. \\
& \left.+\bar{c}_{m n, l}^{(p)} \mathbf{N}_{m n}^{(J)}\left(k_{p l}, \mathbf{r}\right)+\bar{w}_{m n, l}^{(p)} \mathbf{L}_{m n}^{(J)}\left(k_{p l}, \mathbf{r}\right)\right] \\
& -\sum_{J} \sum_{l} \alpha_{p l}^{(J)} \frac{k_{p l}}{\omega \mu_{p s}} \bar{w}_{00, l}^{(p)} \mathbf{L}_{00}^{(J)}\left(k_{p l}, \mathbf{r}\right), \\
\mathbf{E}_{p} & =-i \sum_{n, m} \bar{E}_{m n} \sum_{J} \sum_{l} \alpha_{p l}^{(J)}\left[c_{m n, l}^{(p)} \mathbf{M}_{m n}^{(J)}\left(k_{p l}, \mathbf{r}\right)\right. \\
& \left.+d_{m n, l}^{(p)} \mathbf{N}_{m n}^{(J)}\left(k_{p l}, \mathbf{r}\right)+\frac{\overline{\bar{w}}_{m n, l}^{(p)}}{\lambda_{p l}} \mathbf{L}_{m n}^{(J)}\left(k_{p l}, \mathbf{r}\right)\right] \\
& -i \sum_{J} \sum_{l} \alpha_{p l}^{(J)} \frac{\overline{\bar{w}}_{00, l}^{(p)}}{\lambda_{p l}} \mathbf{L}_{00}^{(J)}\left(k_{p l}, \mathbf{r}\right) .
\end{aligned}
$$

When $p=1,2, \ldots, N-1$, the summation subscript $J$ must take both 1 and 3 to obtain the complete solution of the vector wave functions, since the first-kind spherical Bessel function and the third-kind spherical Bessel function (or sometimes referred to as the first-kind spherical Hankel function) are regular in these regions; however, when $p=N$, the summation subscript $J$ only takes 1 due to the singularity of the second-kind spherical Bessel function (and hence the third-kind spherical Bessel function) at the origin. A detailed derivation for obtaining the expansion coefficients $\alpha_{p l}^{(J)}$ and $k_{p l}$ in (2a) and (2b) can be found in $[22,23]$ and will not be repeated here for simplicity.

2.2. Matching Boundary Conditions. The EM boundary conditions on the interfaces are

$$
\begin{aligned}
\mathbf{E}_{p-1} \times \mathbf{e}_{r} & =\mathbf{E}_{p} \times \mathbf{e}_{r}, \\
\mathbf{H}_{p-1} \times \mathbf{e}_{r} & =\mathbf{H}_{p} \times \mathbf{e}_{r},
\end{aligned}
$$

for $r=a_{p}(p=2,3, \ldots, N)$, and

$$
\begin{aligned}
\mathbf{E}_{1} \times \mathbf{e}_{r} & =\left[\mathbf{E}_{\mathrm{inc}}+\mathbf{E}_{\mathrm{sca}}\right] \times \mathbf{e}_{r}, \\
\mathbf{H}_{1} \times \mathbf{e}_{r} & =\left[\mathbf{H}_{\mathrm{inc}}+\mathbf{H}_{\mathrm{sca}}\right] \times \mathbf{e}_{r},
\end{aligned}
$$

for $r=a_{1}$. In the above equations, $\mathbf{e}_{r}$ is the unit vector of the radial component in spherical coordinate, $\left(\mathbf{E}_{\mathrm{sca}}, \mathbf{H}_{\mathrm{sca}}\right)$ and 
$\left(\mathbf{E}_{\text {inc }}, \mathbf{H}_{\text {inc }}\right)$ are the scattered and incident fields expanded by VSWFs (see (26) and (28) in [23] for details). Based on the boundary conditions, we can obtain the following recurrent formulation:

$$
\overline{\mathbf{T}}_{p-1, p} \cdot \mathbf{X}_{p-1}=\overline{\mathbf{T}}_{p, p} \cdot \mathbf{X}_{p}
$$

where

$$
\begin{aligned}
\overline{\mathbf{T}}_{p, f}=\left[\begin{array}{ll}
\mathscr{A}^{(p, f)} & \overline{\mathscr{A}}^{(p, f)} \\
\mathscr{B}^{(p, f)} & \overline{\mathscr{B}}^{(p, f)} \\
\mathscr{C}^{(p, f)} & \overline{\mathscr{C}}^{(p, f)} \\
\mathscr{D}^{(p, f)} & \overline{\mathscr{D}}^{(p, f)}
\end{array}\right], \\
\mathbf{X}_{p}=\left[\begin{array}{c}
\boldsymbol{\alpha}_{p}^{(1)} \\
\boldsymbol{\alpha}_{p}^{(3)}
\end{array}\right],
\end{aligned}
$$

$$
\begin{aligned}
\overline{\mathbf{T}}_{N, f} & =\left[\begin{array}{ll}
\mathscr{A}^{(N, f)} & \mathbf{0} \\
\mathscr{B}^{(N, f)} & \mathbf{0} \\
\mathscr{C}^{(N, f)} & \mathbf{0} \\
\mathscr{D}^{(N, f)} & \mathbf{0}
\end{array}\right], \\
\mathbf{X}_{N} & =\left[\begin{array}{c}
\boldsymbol{\alpha}_{N}^{(1)} \\
\mathbf{0}
\end{array}\right],
\end{aligned}
$$

$$
\text { for } p=N \text {. }
$$

The matrix elements are defined as

$$
\begin{aligned}
& A_{m n, l}^{(p, f)}=\frac{k_{0}}{k_{p l}} \frac{\psi_{n}^{\prime}\left(k_{p l} a_{f}\right)}{\psi_{n}^{\prime}\left(k_{0} a_{f}\right)} d_{m n, l}^{(p)}+\frac{k_{0}}{k_{p s}^{2} a_{f}} \frac{\psi_{n}\left(k_{p l} a_{f}\right)}{\psi_{n}^{\prime}\left(k_{0} a_{f}\right)} \overline{\bar{w}}_{m n, l}^{(p)}, \\
& \bar{A}_{m n, l}^{(p, f)}=\frac{k_{0}}{k_{p l}} \frac{\xi_{n}^{\prime}\left(k_{p l} a_{f}\right)}{\psi_{n}^{\prime}\left(k_{0} a_{f}\right)} d_{m n, l}^{(p)}+\frac{k_{0}}{k_{p s}^{2} a_{f}} \frac{\xi_{n}\left(k_{p l} a_{f}\right)}{\psi_{n}^{\prime}\left(k_{0} a_{f}\right)} \overline{\bar{w}}_{m n, l}^{(p)}, \\
& B_{m n, l}^{(p, f)}=\frac{k_{0}}{k_{p l}} \frac{\psi_{n}\left(k_{p l} a_{f}\right)}{\psi_{n}\left(k_{0} a_{f}\right)} c_{m n, l}^{(p)}, \\
& \bar{B}_{m n, l}^{(p, f)}=\frac{k_{0}}{k_{p l}} \frac{\xi_{n}\left(k_{p l} a_{f}\right)}{\psi_{n}\left(k_{0} a_{f}\right)} c_{m n, l}^{(p)}, \\
& C_{m n, l}^{(p, f)}=\frac{\mu_{0}}{\mu_{p s}} \frac{\psi_{n}\left(k_{p l} a_{f}\right)}{\psi_{n}\left(k_{0} a_{f}\right)} \bar{d}_{m n, l}^{(p)}, \\
& \bar{C}_{m n, l}^{(p, f)}=\frac{\mu_{0}}{\mu_{p s}} \frac{\xi_{n}\left(k_{p l} a_{f}\right)}{\psi_{n}\left(k_{0} a_{f}\right)} \bar{d}_{m n, l}^{(p)},
\end{aligned}
$$

$$
\begin{aligned}
D_{m n, l}^{(p, f)}= & \frac{\mu_{0}}{\mu_{p s}} \frac{\psi_{n}^{\prime}\left(k_{p l} a_{f}\right)}{\psi_{n}^{\prime}\left(k_{0} a_{f}\right)} \bar{c}_{m n, l}^{(p)} \\
& +\frac{\mu_{0}}{k_{p l} \mu_{p s} a_{f}} \frac{\psi_{n}\left(k_{p l} a_{f}\right)}{\psi_{n}^{\prime}\left(k_{0} a_{f}\right)} \bar{w}_{m n, l}^{(p)}, \\
\bar{D}_{m n, l}^{(p, f)}= & \frac{\mu_{0}}{\mu_{p s}} \frac{\xi_{n}^{\prime}\left(k_{p l} a_{f}\right)}{\psi_{n}^{\prime}\left(k_{0} a_{f}\right)} \bar{c}_{m n, l}^{(p)} \\
& +\frac{\mu_{0}}{k_{p l} \mu_{p s} a_{f}} \frac{\xi_{n}\left(k_{p l} a_{f}\right)}{\psi_{n}^{\prime}\left(k_{0} a_{f}\right)} \bar{w}_{m n, l}^{(p)} .
\end{aligned}
$$

After a careful comparison of (7) and (6) in [23], we observe that, by adding the gyromagnetic tensors, additional terms are introduced into the matrix elements of $D_{m n, l}^{(p, f)}$ and $\bar{D}_{m n, l}^{(p, f)}$. In (7), $\psi_{n}(z)$ and $\xi_{n}(z)$ are the Riccati-Bessel functions given by

$$
\begin{aligned}
& \psi_{n}(z)=z j_{n}(z), \\
& \xi_{n}(z)=z h_{n}^{(1)}(z),
\end{aligned}
$$

with $j_{n}(z)$ and $h_{n}^{(1)}(z)$ being the spherical Bessel functions of the first and the third kind, respectively. According to the recurrence relations in (5), the expansion coefficients $\mathbf{X}_{1}$ in Region-1 can be related to $\mathbf{X}_{N}$ in Region- $N$ as

$$
\mathbf{X}_{1}=\left[\begin{array}{l}
\boldsymbol{\alpha}_{1}^{(1)} \\
\boldsymbol{\alpha}_{1}^{(3)}
\end{array}\right]=\mathscr{T} \cdot \mathbf{X}_{N},
$$

where $\mathscr{T}=\overline{\mathbf{T}}_{1,2}^{-1} \cdot \overline{\mathbf{T}}_{2,2} \cdots \overline{\mathbf{T}}_{N-1, N}^{-1} \cdot \overline{\mathbf{T}}_{N, N}$. Then we apply the EM boundary condition in (4a) and (4b) at $r=a_{1}$ to solve a scattering problem as

$$
\begin{gathered}
{\left[\begin{array}{ll}
\bar{\Lambda} & 0 \\
0 & \Lambda
\end{array}\right]\left[\begin{array}{l}
\mathbf{a} \\
\mathbf{b}
\end{array}\right]+\left[\begin{array}{ll}
\mathscr{A}^{(1,1)} & \overline{\mathscr{A}}^{(1,1)} \\
\mathscr{B}^{(1,1)} & \overline{\mathscr{B}}^{(1,1)}
\end{array}\right]\left[\begin{array}{l}
\boldsymbol{\alpha}_{1}^{(1)} \\
\boldsymbol{\alpha}_{1}^{(3)}
\end{array}\right]=\left[\begin{array}{l}
\mathbf{p} \\
\mathbf{q}
\end{array}\right],} \\
{\left[\begin{array}{ll}
\Lambda & 0 \\
0 & \bar{\Lambda}
\end{array}\right]\left[\begin{array}{l}
\mathbf{a} \\
\mathbf{b}
\end{array}\right]+\left[\begin{array}{ll}
\mathscr{C}^{(1,1)} & \overline{\mathscr{C}}^{(1,1)} \\
\mathscr{D}^{(1,1)} & \overline{\mathscr{D}}^{(1,1)}
\end{array}\right]\left[\begin{array}{l}
\boldsymbol{\alpha}_{1}^{(1)} \\
\boldsymbol{\alpha}_{1}^{(3)}
\end{array}\right]=\left[\begin{array}{l}
\mathbf{p} \\
\mathbf{q}
\end{array}\right] .}
\end{gathered}
$$

In (10a) and (10b), $[\mathbf{a}, \mathbf{b}]^{T}$ and $[\mathbf{p}, \mathbf{q}]^{T}$ are the scattering and incident coefficients (see detailed expressions in [23]). The matrices $\Lambda$ and $\bar{\Lambda}$ are expressed as [20]

$$
\begin{aligned}
& \Lambda_{m n, u v}=\frac{\xi_{n}\left(x_{1}\right)}{\psi_{n}\left(x_{1}\right)} \delta_{n v} \delta_{m u}, \\
& \bar{\Lambda}_{m n, u v}=\frac{\xi_{n}^{\prime}\left(x_{1}\right)}{\psi_{n}^{\prime}\left(x_{1}\right)} \delta_{n v} \delta_{m u},
\end{aligned}
$$

where $x_{1}=k_{0} a_{1}$. By substituting (9) into (10a) and (10b), the scattering coefficients $a_{m n}$ and $b_{m n}$ and the expansion 
coefficients $\alpha_{N}$ for Region- $N$ can all be solved numerically. After obtaining $\mathbf{X}_{N}$, the field expansion coefficients for internal layers (from Region- $(N-1)$ to Region-1) can be readily calculated by applying the inward to outward recurrence procedure.

When $r \rightarrow \infty$, the asymptotic forms of the third-kind VSWFs are expressed as [34]

$$
\begin{aligned}
& \mathbf{M}_{m n}^{(3)}\left(k_{0}, \mathbf{r}\right) \\
& =(-i)^{n}\left[\pi_{m n}(\cos \theta) \mathbf{e}_{\theta}+i \tau_{m n}(\cos \theta) \mathbf{e}_{\phi}\right] \frac{e^{i k_{0} r}}{k_{0} r} e^{i m \phi}, \\
& \mathbf{N}_{m n}^{(3)}\left(k_{0}, \mathbf{r}\right) \\
& =(-i)^{n}\left[\tau_{m n}(\cos \theta) \mathbf{e}_{\theta}+i \pi_{m n}(\cos \theta) \mathbf{e}_{\phi}\right] \frac{e^{i k_{0} r}}{k_{0} r} e^{i m \phi},
\end{aligned}
$$

where $\mathbf{e}_{\theta}$ and $\mathbf{e}_{\phi}$ are the unit vectors of the $\theta$ and $\phi$ components in the spherical coordinate, respectively, and

$$
\begin{aligned}
\pi_{m n}(\cos \theta) & =\frac{m}{\sin \theta} P_{n}^{m}(\cos \theta), \\
\tau_{m n}(\cos \theta) & =\frac{\mathrm{d} P_{n}^{m}(\cos \theta)}{\mathrm{d} \theta} .
\end{aligned}
$$

Consequently, the RCS of the multilayered gyrotropic scatterer can be calculated by using the obtained scattering coefficients as

$$
\begin{aligned}
\sigma & =\lim _{r \rightarrow \infty} 4 \pi r^{2} \frac{\left|\mathbf{E}_{\text {sca }}\right|^{2}}{\left|\mathbf{E}_{\text {inc }}\right|^{2}} \\
& =\frac{\lambda^{2}}{\pi} \mid \sum_{n, m} \bar{E}_{m n}\left\{\left[a_{m n} \pi_{m n}(\cos \theta)+b_{m n} \tau_{m n}(\cos \theta)\right] \mathbf{e}_{\theta}\right. \\
& \left.+i\left[a_{m n} \tau_{m n}(\cos \theta)+b_{m n} \pi_{m n}(\cos \theta)\right] \mathbf{e}_{\phi}\right\}\left.e^{i m \phi}\right|^{2}
\end{aligned}
$$

\section{Numerical Results and Discussions}

The incident plane wave is assumed to be propagating along the positive $z$ direction, where the electric field is polarized in the $x$ direction with unit amplitude. The size parameters of the multilayered sphere are represented as $x_{p}=k_{0} a_{p}=$ $2 \pi a_{p} / \lambda(p=1,2, \ldots, N)$, which are essentially the electrical dimensions with respect to the incident wavelength $\lambda$. In the practical computations, the summation index $n$ in (2a) and (2b) should be truncated at $n_{c}$. As suggested in Appendix A in [32] and (8) in [35], $n_{c}$ can be chosen to be

$$
n_{c}=x+4 x^{1 / 3}+2 \text {, }
$$

where $x$ denotes the size parameter of the spherical scatterer. In this paper, $x$ is uniformly chosen as $x=x_{1}=k_{0} a_{1}$ to make the matrices in (5) have the same dimensions. The linear equations (10a) and (10b) are solved independently of each $m \in\left[-n_{c}, n_{c}\right]$ due to the Kronecker symbols $\delta_{m p}$ appearing in (1la) and (11b). In the following discussions, the numerical

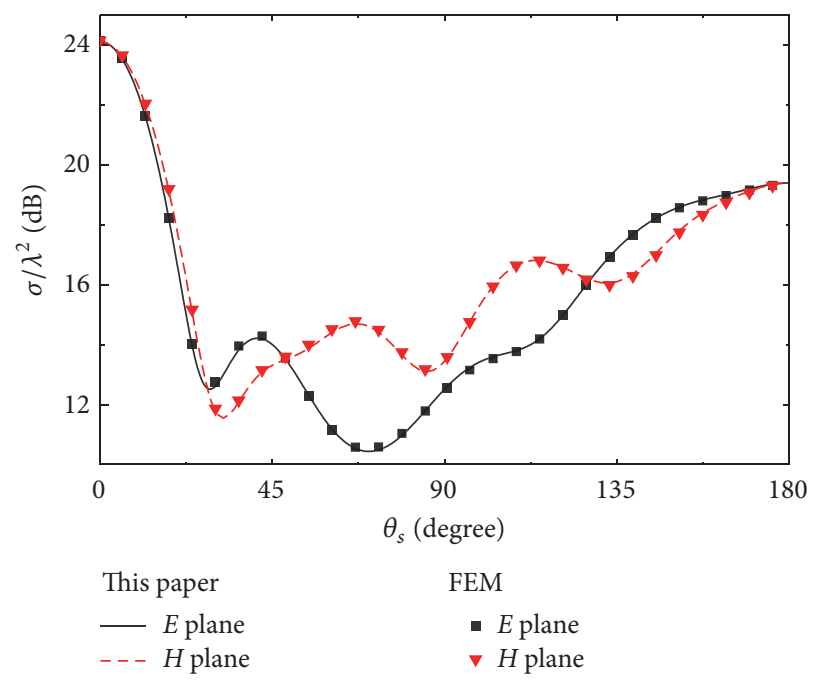

FIGURE 2: Normalized RCS of a 2-layered gyrotropic sphere versus scattering angle $\theta_{s}$, where the electric dimensions are chosen as $x_{1}=$ $2 \pi$ and $x_{2}=1.5 \pi$. The permittivity and permeability tensor elements are assumed to be $\epsilon_{1 \mathrm{~s}}=\epsilon_{2 s}=\epsilon_{0}, \epsilon_{1 r}=1, \epsilon_{2 r}=2$, and $\epsilon_{1 k}=\epsilon_{2 k}=1+i$ and $\mu_{1 s}=\mu_{2 s}=\mu_{0}, \mu_{1 r}=1, \mu_{2 r}=2$, and $\mu_{1 k}=\mu_{2 k}=1+0.5 i$.

results of RCS are normalized by the square of the incident wavelength $\left(\lambda^{2}\right)$.

The convergence criteria (15) are commonly used for homogeneous spherical scatterers $[35,36]$. As for the gyrotropic case considered in this work, the validation of truncation number $n_{c}$ has been checked by computing the relative error of truncated series of the scattering efficiency factor $Q_{\text {sca }}=\left(4 / x_{1}^{2}\right) \sum_{n, m}\left(\left|a_{m n}\right|^{2}+\left|b_{m n}\right|^{2}\right)[20,36]$. Explicitly, the relative error is defined as $\varepsilon(N)=\left|Q_{\text {sca }}^{(N)}-Q_{\text {sca }}\right| /\left|Q_{\text {sca }}\right|$, where $Q_{\mathrm{sca}}^{(N)}$ is the truncated series and $Q_{\mathrm{sca}}$ is the converged value (calculated for a sufficiently large $N$ ). Four cases with different electrical dimensions are depicted in Figure 4. It can be seen that the truncated series converge well when the summation index $n$ is truncated at the value given by (15), and the convergence accuracy is at least $10^{-5}$.

To verify the correctness of the formulations derived for the multilayered gyrotropic sphere in this paper, two numerical experiments are conducted. First, the normalized RCS values of a 2-layered gyrotropic sphere are compared with that computed from the full-wave finite element method (FEM) simulation, as depicted in Figure 2. The normalized root mean square deviations (NRMSD) for $E$-plane (xoz) and $H$ - plane $(y o z)$ are 0.0041 and 0.0052 , respectively. In the FEM simulation, a mesh with 53294 tetrahedral elements has been used and the basis functions are constructed from polynomials of order 3 . Second, the gyrotropic case is reduced to the uniaxial case (i.e., $\mu_{p \kappa}=0$ and $\epsilon_{p \kappa}=0$, for $p=$ $1,2, \ldots, N)$, so that a direct comparison can be made with the published results (Figure 4 in [33]), as shown in Figure 3. The NRMSD for $E$ - and $H$-planes are 0.007 and 0.0099 , respectively. In both cases, excellent agreements of the RCS values are achieved in both $E$-plane and $H$-plane, confirming the correctness of the newly derived formulations. 


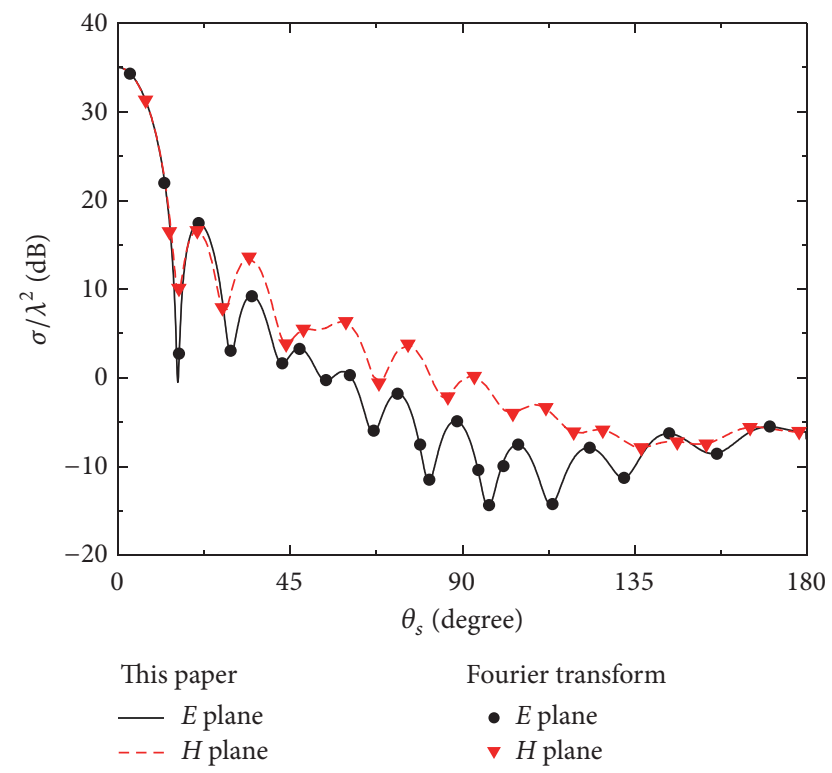

Figure 3: Normalized RCS of a 6-layered uniaxial anisotropic sphere versus scattering angle $\theta_{s}$, where the electric dimensions are chosen as $x_{1}=4.1 \pi, x_{2}=4 \pi, x_{3}=3.9 \pi, x_{4}=3.8 \pi, x_{5}=3.7 \pi$, and $x_{6}=3.6 \pi$. The permittivity and permeability tensor elements are assumed to be $\epsilon_{1 s} \epsilon_{1 r}=\epsilon_{3 s} \epsilon_{3 r}=\epsilon_{5 s} \epsilon_{5 r}=\epsilon_{2 s}=\epsilon_{4 s}=\epsilon_{6 s}=(3+0.15 i) \epsilon_{0}$ and $\epsilon_{2 s} \epsilon_{2 r}=\epsilon_{4 s} \epsilon_{4 r}=\epsilon_{6 s} \epsilon_{6 r}=\epsilon_{1 s}=\epsilon_{3 s}=\epsilon_{5 s}=(2+0.1 i) \epsilon_{0}$ and $\mu_{1 s} \mu_{1 r}=\mu_{3 s} \mu_{3 r}=\mu_{5 s} \mu_{5 r}=\mu_{2 s}=\mu_{4 s}=\mu_{6 s}=(1+0.05 i) \mu_{0}$, $\mu_{2 s} \mu_{2 r}=\mu_{4 s} \mu_{4 r}=\mu_{6 s} \mu_{6 r}=\mu_{1 s}=\mu_{3 s}=\mu_{5 s}=(2+0.1 i) \mu_{0}$.

In the following, three specific cases will be presented to further investigate the scattering characteristic of the multilayered gyrotropic sphere.

In Figure 5, the bistatic RCS of a 2-layered lossless gyrotropic sphere with small-size parameters is presented, where two cases of different EM parameter settings are studied comparatively. The RCS results are shown in Figures 5(a) and 5(b). We observe that if the permittivity and permeability tensors in each layer satisfy the following conditions,

$$
\begin{aligned}
& \frac{\epsilon_{p s}}{\epsilon_{0}}=\frac{\mu_{p s}}{\mu_{0}}, \\
& \epsilon_{p r}=\mu_{p r}, \\
& \epsilon_{p \kappa}=\mu_{p \kappa},
\end{aligned}
$$

the RCS in $E$-plane and $H$-plane coincide with each other. More importantly, a sharp RCS reduction in the backscattering direction can be obviously observed. Therefore, this property may have potential applications in stealth or cloaking design.

To further understand this scattering property, another 4layered lossy gyrotropic sphere with medium-size parameters is considered and the bistatic RCS values are depicted in Figure 6. In this case, the parameters are chosen based on (16), and the property presented in Figure 5(b) still holds for this case. It can be possibly concluded that if the parameters in each layer satisfy (16), the RCS in the backward direction can

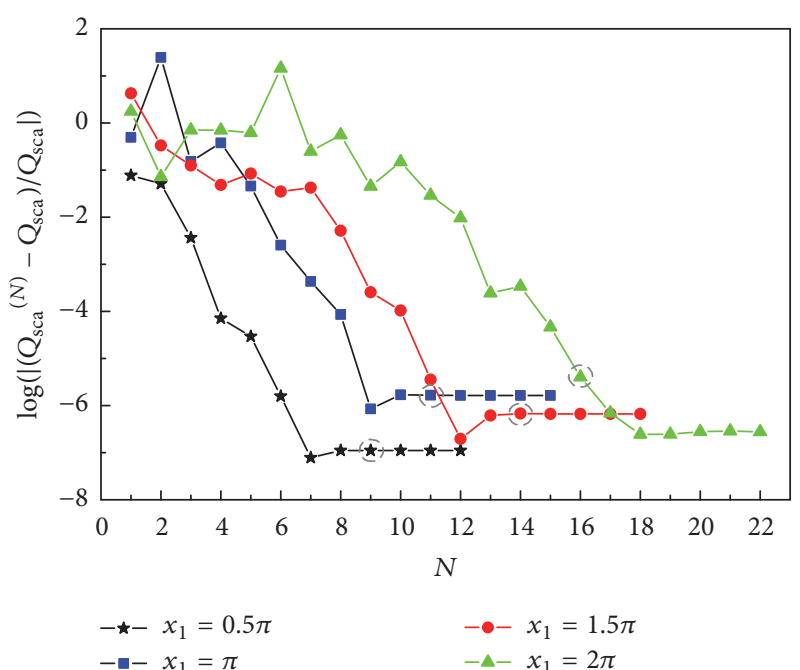

FIGURE 4: Logarithm values of the relative error $\varepsilon(N)$ versus the truncation number $N$ for a 2-layered gyrotropic sphere with four different electric dimensions. The EM parameters considered here are the same as those in Figure 2 and the size parameters $x_{2}$ are equal to $0.75 x_{1}$. The dashed circles indicate the positions of truncation numbers given in (15).

be made too weak to be detected, no matter for lossless or lossy anisotropic materials.

In Figure 7, the bistatic RCS of a 2-layered lossless gyrotropic sphere with large-size parameters is presented. The EM parameters are configured specifically to give another insight of the scattering property. From Figure 7, it can be observed that the RCS becomes more oscillatory than those depicted in Figures 5 and 6 . When the scattering angle increases from $0^{\circ}$ to $180^{\circ}$, the RCS decreases first and then increases to a high level in both $E$-plane and $H$-plane.

\section{Conclusions}

The analytical solutions of the EM scattering by a radially multilayered gyrotropic sphere are studied in this paper. First, by applying the completeness and noncoplanar properties of the VSWFs, theoretical formulations of this scattering problem have been derived. Second, we compare the numerical results of RCS values with those obtained from the full-wave simulation or from the literature to verify the validity and correctness of the proposed method. Third, specific cases are investigated to reveal some interesting physical insights of the scattering property of the multilayered gyrotropic sphere. It is found that if the gyrotropic tensors are configured properly, the RCS in the backward scattering direction can be suppressed or enhanced significantly.

\section{Conflicts of Interest}

The authors declare that they have no conflicts of interest. 


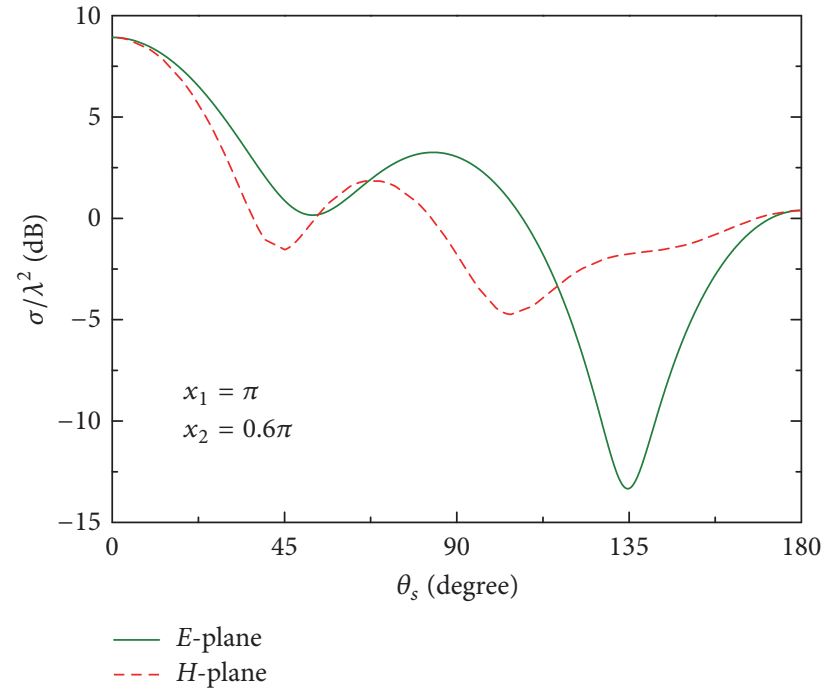

(a) Case 1

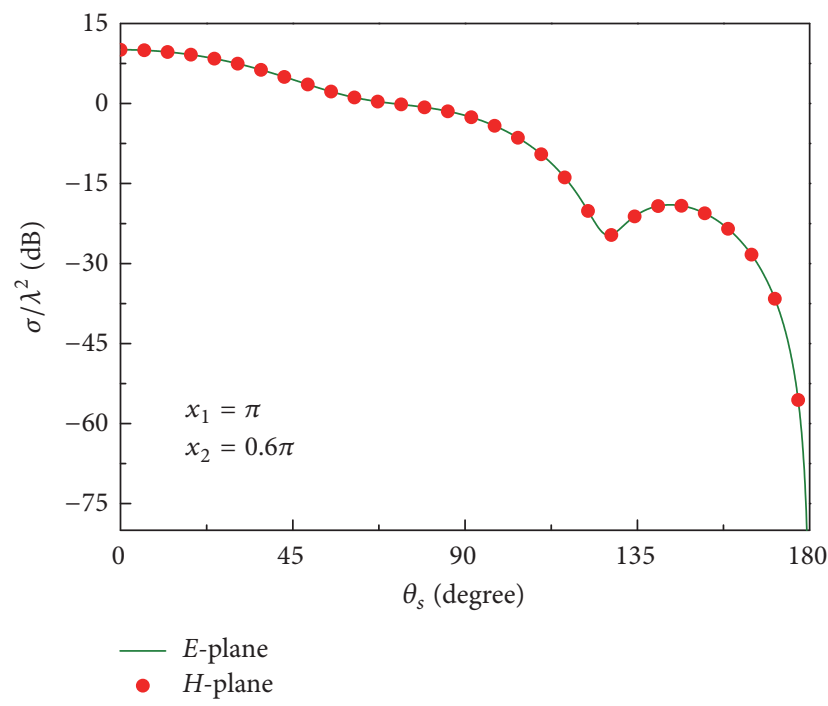

(b) Case 2

FiguRE 5: Normalized RCS of a 2-layered lossless gyrotropic sphere versus scattering angle $\theta_{s}$ in $E$-plane and $H$-plane. The gyrotropic tensors in each layer are selected as (a) $\epsilon_{1 s}=4 \epsilon_{0}, \mu_{1 s}=2 \mu_{0}, \epsilon_{2 s}=2 \epsilon_{0}, \mu_{2 s}=4 \mu_{0}, \epsilon_{p r}=\mu_{p r}=0.5, \epsilon_{p k}=\mu_{p k}=0.25$, and $p=1,2$; (b) $\epsilon_{1 s}=2 \epsilon_{0}$, $\mu_{1 s}=2 \mu_{0}, \epsilon_{2 s}=4 \epsilon_{0}, \mu_{2 s}=4 \mu_{0}, \epsilon_{p r}=\mu_{p r}=0.5, \epsilon_{p k}=\mu_{p k}=0.25$, and $p=1,2$.

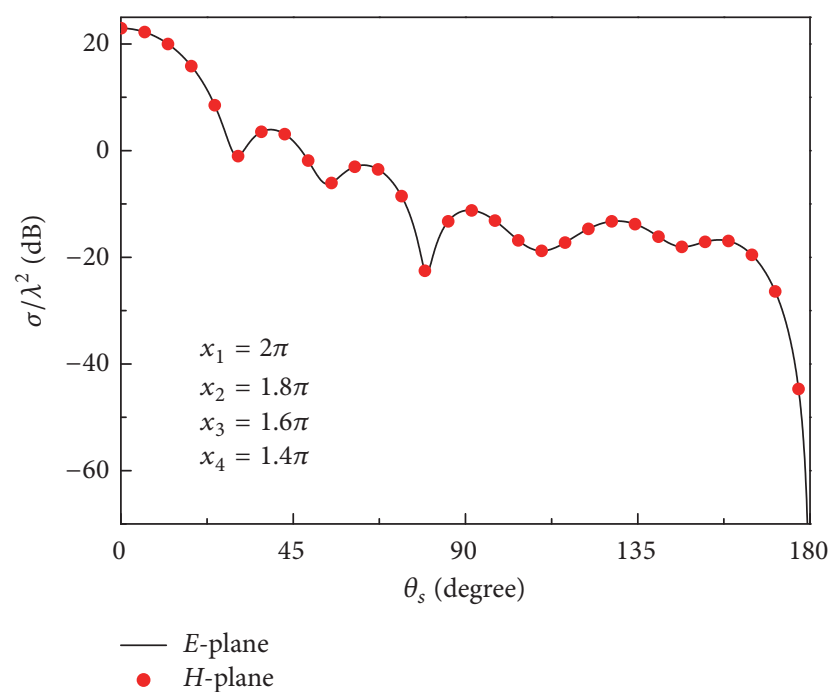

Figure 6: Normalized RCS of a 4-layered lossy gyrotropic sphere versus scattering angle $\theta_{s}$ in $E$-plane and $H$-plane. The gyrotropic tensors in each layer are selected as $\epsilon_{p s}=(5+0.2 i) \epsilon_{0}, \mu_{p s}=(5+$ $0.2 i) \mu_{0}, \epsilon_{p s} \epsilon_{p k}=\epsilon_{0}, \mu_{p s} \mu_{p k}=\mu_{0}$ (where $\left.p=1,2,3,4\right), \epsilon_{1 s} \epsilon_{1 r}=(3+$ $0.1 i) \epsilon_{0}, \mu_{1 s} \mu_{1 r}=(3+0.1 i) \mu_{0}, \epsilon_{2 s} \epsilon_{2 r}=(5+0.1 i) \epsilon_{0}, \mu_{2 s} \mu_{2 r}=(5+0.1 i) \mu_{0}$, $\epsilon_{3 s} \epsilon_{3 r}=(7+0.1 i) \epsilon_{0}, \mu_{3 s} \mu_{3 r}=(7+0.1 i) \mu_{0}, \epsilon_{4 s} \epsilon_{4 r}=(9+0.1 i) \epsilon_{0}$, and $\mu_{4 s} \mu_{4 r}=(9+0.1 i) \mu_{0}$.

\section{Acknowledgments}

The authors are grateful to the useful discussions with Mr. Chao Wan at the National University of Singapore. L. Cao acknowledges the financial support from Special Talent Program at University of Electronic Science and Technology of China (UESTC). This is in memory of Professor Joshua Le-Wei Li, and taking this opportunity, the authors wish to

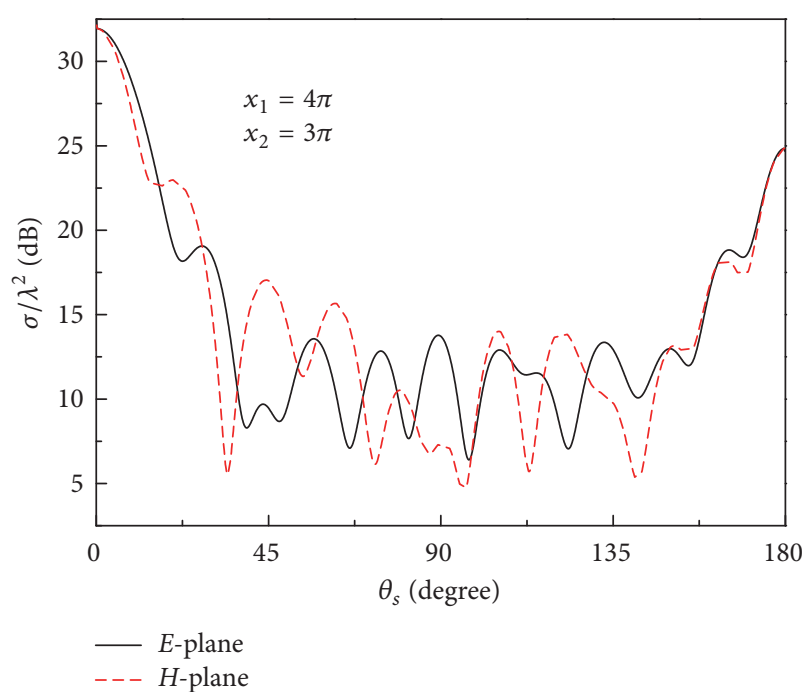

FIGURE 7: Normalized RCS of a 2-layered lossless gyrotropic sphere versus scattering angle $\theta_{s}$ in $E$-plane and $H$-plane. The gyrotropic tensors in each layer are selected as $\epsilon_{1 s}=2 \epsilon_{0}, \epsilon_{1 r}=2, \epsilon_{1 k}=0.5$, $\mu_{1 s}=4 \mu_{0}, \mu_{1 r}=0.5$, and $\mu_{1 k}=0.25 ; \epsilon_{2 s}=4 \epsilon_{0}, \epsilon_{2 r}=0.5, \epsilon_{2 k}=0.25$, $\mu_{2 s}=2 \mu_{0}, \mu_{2 r}=2$, and $\mu_{2 k}=0.5$.

express their genuine gratitude to him for his kind and patient guidance.

\section{References}

[1] J. Luo, W. Lu, Z. Hang et al., "Arbitrary control of electromagnetic flux in inhomogeneous anisotropic media with near-zero index," Physical Review Letters, vol. 112, no. 7, Article ID 073903, 2014. 
[2] Z.-J. Li, H.-Y. Li, and Z.-S. Wu, "Analysis of electromagnetic scattering by uniaxial anisotropic bispheres," Journal of the Optical Society of America A: Optics and Image Science, and Vision, vol. 28, no. 2, pp. 118-125, 2011.

[3] Y. Petrov, "Anisotropic spherical head model and its application to imaging electric activity of the brain," Physical Review E Statistical, Nonlinear, and Soft Matter Physics, vol. 86, no. 1, Article ID 011917, 2012.

[4] K. Sainath and F. L. Teixeira, “Tensor Green's function evaluation in arbitrarily anisotropic, layered media using complexplane Gauss-Laguerre quadrature," Physical Review E - Statistical, Nonlinear, and Soft Matter Physics, vol. 89, no. 5, Article ID 053303, 2014.

[5] T.-J. Guo, T.-F. Li, M. Yang et al., "Nonreciprocal optical diffraction by a single layer of gyromagnetic cylinders," Optics Express, vol. 22, no. 1, pp. 537-546, 2014.

[6] R. C. Haussman and M. Deserno, "Effective field theory of thermal Casimir interactions between anisotropic particles," Physical Review E - Statistical, Nonlinear, and Soft Matter Physics, vol. 89, no. 6, Article ID 062102, 2014.

[7] J. Christensen and F. J. G. de Abajo, "Anisotropic metamaterials for full control of acoustic waves," Physical Review Letters, vol. 108, no. 12, Article ID 124301, 2012.

[8] Z. Wu, M. Levy, V. J. Fratello, and A. M. Merzlikin, "Gyrotropic photonic crystal waveguide switches," Applied Physics Letters, vol. 96, no. 5, Article ID 051125, 2010.

[9] N. K. Dissanayake, M. Levy, A. A. Jalali, and V. J. Fratello, "Gyrotropic band gap optical sensors," Applied Physics Letters, vol. 96, no. 18, Article ID 181105, 2010.

[10] G. W. Ford, J. K. Furdyna, and S. A. Werner, "Electromagnetic excitations of a small gyrotropic sphere," Physical Review B, vol. 12, no. 4, pp. 1452-1465, 1975.

[11] R.-J. Tarento, K.-H. Bennemann, P. Joyes, and J. Van de Walle, "Mie scattering of magnetic spheres," Physical Review E Statistical, Nonlinear, and Soft Matter Physics, vol. 69, no. 2, Article ID 026606, pp. 1-26606, 2004.

[12] G. W. Ford and S. A. Werner, "Scattering and absorption of electromagnetic waves by a gyrotropic sphere," Physical Review $B$, vol. 18, no. 12, pp. 6752-6769, 1978.

[13] Y.-L. Geng, X.-B. Wu, L.-W. Li, and B.-R. Guan, "Mie scattering by a uniaxial anisotropic sphere," Physical Review E, vol. 70, no. 5, Article ID 056609, 2004.

[14] Y.-L. Geng, X.-B. Wu, and L.-W. Li, "Characterization of electromagnetic scattering by a plasma anisotropic spherical shell,' IEEE Antennas and Wireless Propagation Letters, vol. 3, no. 1, pp. 100-103, 2004.

[15] Y.-L. Geng, C.-W. Qiu, and N. Yuan, "Exact solution to electromagnetic scattering by an impedance sphere coated with a uniaxial anisotropic layer," Institute of Electrical and Electronics Engineers. Transactions on Antennas and Propagation, vol. 57, no. 2, pp. 572-576, 2009.

[16] A. Eroglu and J. K. Lee, "Dyadic green's functions for an electrically gyrotropic medium," Progress In Electromagnetics Research, vol. 58, pp. 223-241, 2006.

[17] A. Eroglu and J. K. Lee, "Simplified formulation of dyadic green's functions and their duality relations for general anisotropic media," Progress In Electromagnetics Research, vol. 77, pp. 391408, 2007.

[18] L.-W. Li, N.-H. Lim, W.-Y. Yin, and J.-A. Kong, "Eigenfunctional expansion of dyadic Green's functions in gyrotropic media using cylindrical vector wave functions," Progress in Electromagnetics Research, vol. 43, pp. 101-121, 2003.
[19] B. Stout, M. Nevière, and E. Popov, "T matrix of the homogeneous anisotropic sphere: applications to orientation-averaged resonant scattering," J. Opt. Soc. Am. A, vol. 24, no. 4, pp. 11201130, 2007.

[20] Z. Lin and S. T. Chui, "Electromagnetic scattering by optically anisotropic magnetic particle," Physical Review E, vol. 69, no. 5, Article ID 056614, 2004.

[21] J. L. Li and W.-L. Ong, "A new solution for characterizing electromagnetic scattering by a gyroelectric sphere," Institute of Electrical and Electronics Engineers. Transactions on Antennas and Propagation, vol. 59, no. 9, pp. 3370-3378, 2011.

[22] J. L.-W. Li, W.-L. Ong, and K. H. R. Zheng, "Anisotropic scattering effects of a gyrotropic sphere characterized using the T-matrix method," Physical Review E - Statistical, Nonlinear, and Soft Matter Physics, vol. 85, no. 3, Article ID 036601, 2012.

[23] L. Cao, J. L. Li, and J. Hu, "A new T-matrix formulation for electromagnetic scattering by a radially multilayered gyroelectric sphere," Institute of Electrical and Electronics Engineers. Transactions on Antennas and Propagation, vol. 61, no. 2, pp. 836-842, 2013.

[24] C.-W. Qiu, L.-W. Li, Q. Wu, and T.-S. Yeo, "Field representations in general gyrotropic media in spherical coordinates," IEEE Antennas and Wireless Propagation Letters, vol. 4, no. 1, pp. 467470, 2005.

[25] C.-W. Qiu, L.-W. Li, T.-S. Yeo, and S. Zouhdi, "Scattering by rotationally symmetric anisotropic spheres: Potential formulation and parametric studies," Physical Review E - Statistical, Nonlinear, and Soft Matter Physics, vol. 75, no. 2, Article ID 026609, 2007.

[26] C.-W. Qiu, S. d. Zouhdi, and A. Razek, "Modified spherical wave functions with anisotropy ratio: application to the analysis of scattering by multilayered anisotropic shells," Institute of Electrical and Electronics Engineers. Transactions on Antennas and Propagation, vol. 55, no. 12, pp. 3515-3523, 2007.

[27] C.-W. Qiu and B. Luk'yanchuk, "Peculiarities in light scattering by spherical particles with radial anisotropy," Journal of the Optical Society of America A, vol. 25, no. 7, pp. 1623-1628, 2008.

[28] C.-W. Qiu, L. Hu, X. Xu, and Y. Feng, "Spherical cloaking with homogeneous isotropic multilayered structures," Physical Review E - Statistical, Nonlinear, and Soft Matter Physics, vol. 79, no. 4, Article ID 047602, 2009.

[29] C.-W. Qiu, A. Novitsky, H. Ma, and S. Qu, "Electromagnetic interaction of arbitrary radial-dependent anisotropic spheres and improved invisibility for nonlinear-transformation-based cloaks," Physical Review E - Statistical, Nonlinear, and Soft Matter Physics, vol. 80, no. 1, Article ID 016604, 2009.

[30] C.-W. Qiu, L. Gao, J. D. Joannopoulos, and M. Soljacic, "Light scattering from anisotropic particles: propagation, localization, and nonlinearity," Laser \& Photonics Reviews, 2010.

[31] D. Sarkar and N. J. Halas, "General vector basis function solution of Maxwell's equations," Physical Review E. Statistical, Nonlinear, and Soft Matter Physics, vol. 56, no. 1, part B, pp. 11021112, 1997.

[32] C. F. Bohren and D. R. Huffman, Absorption and Scattering of Light by Small Particles, John Wiley \& Sons, NY, USA, 1998.

[33] Y.-L. Geng, S.-X. Guo, and L.-W. Li, "EM scattering by radially multilayered uniaxial anisotropic spheres," in Proceedings of the Asia Pacific Microwave Conference 2009, APMC 2009, pp. 669672, sgp, December 2009.

[34] Y.-L. Xu, "Electromagnetic scattering by an aggregate of spheres: far field," Applied Optics, vol. 36, no. 36, pp. 9496-9508, 1997. 
[35] W. J. Wiscombe, "Improved mie scattering algorithms," Applied Optics, vol. 19, no. 9, pp. 1505-1509, 1980.

[36] J. R. Allardice and E. C. Le Ru, "Convergence of mie theory series: criteria for far-field and near-field properties," Applied Optics, vol. 53, no. 31, pp. 7224-7229, 2014. 


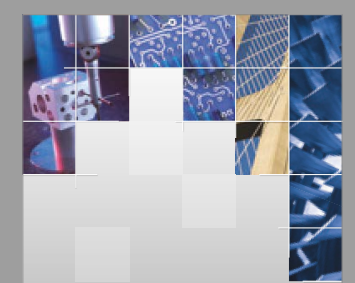

\section{Enfincering}
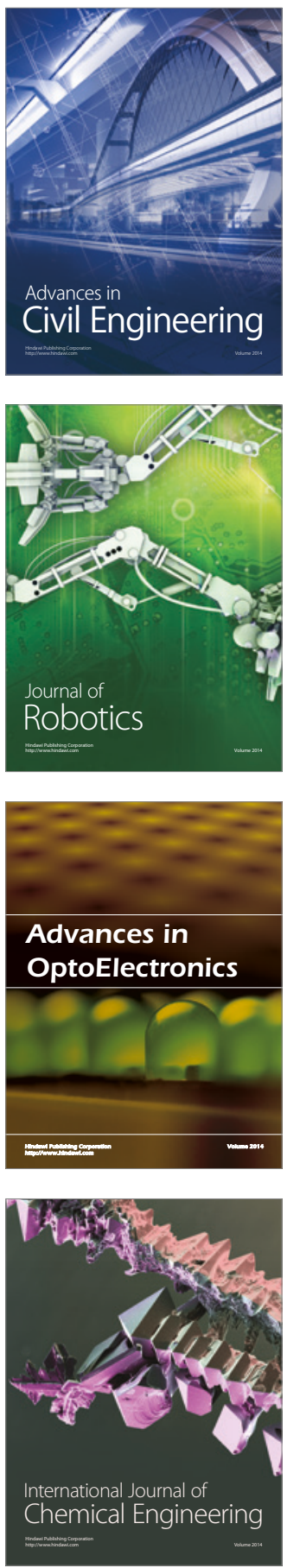

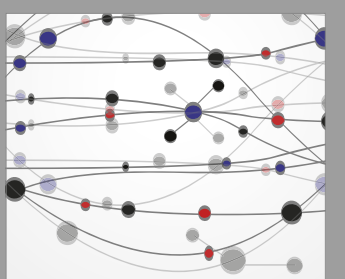

The Scientific World Journal



\section{Hindawi}

Submit your manuscripts at

https://www.hindawi.com
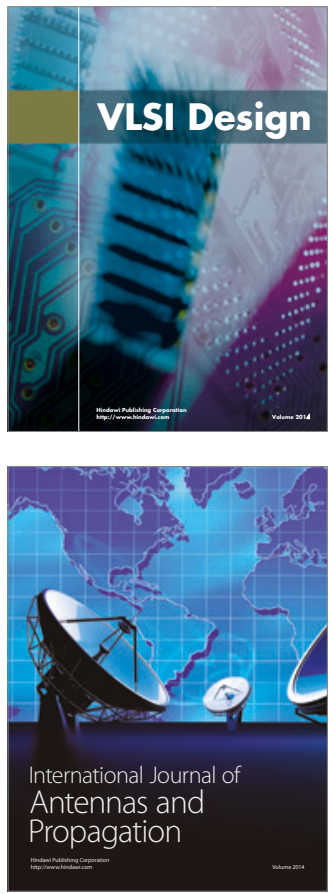

\section{Rotating}

Machinery
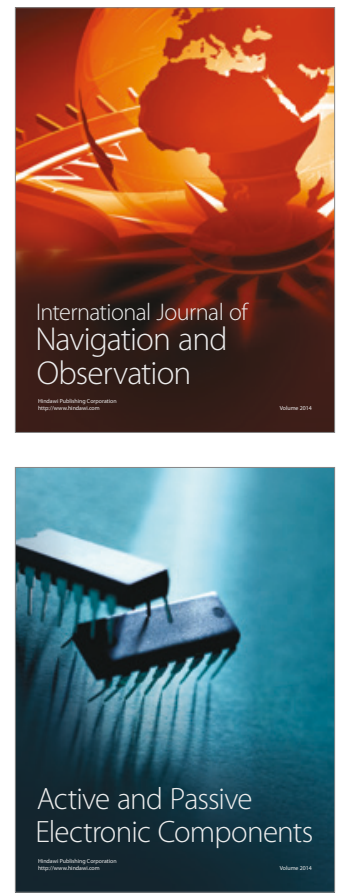
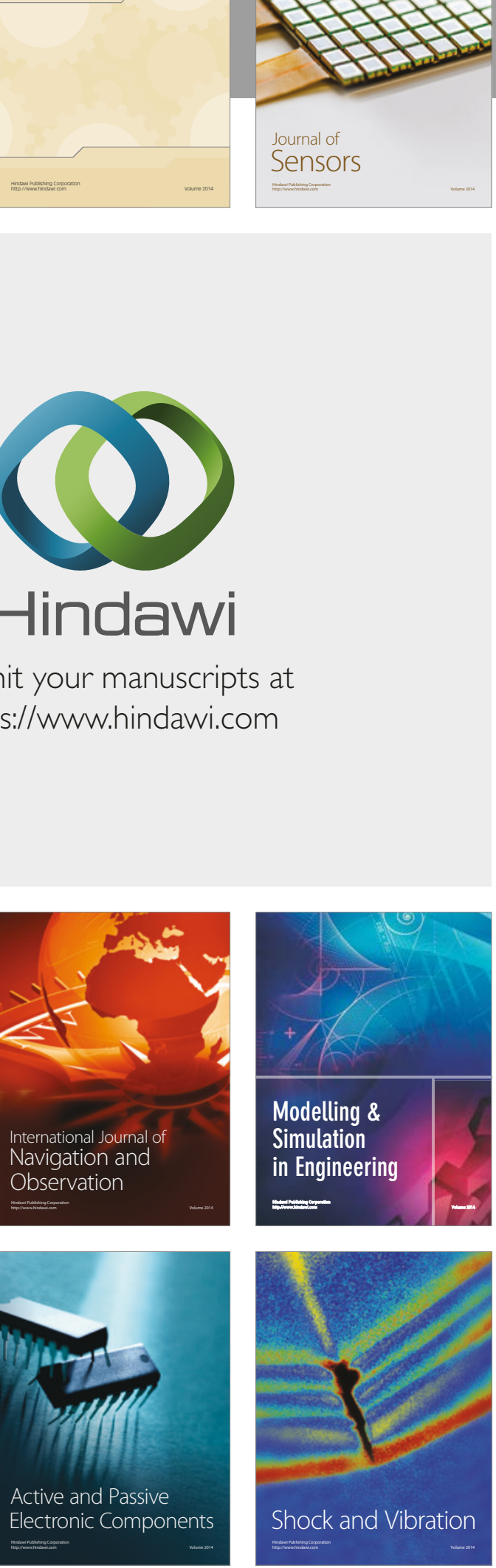
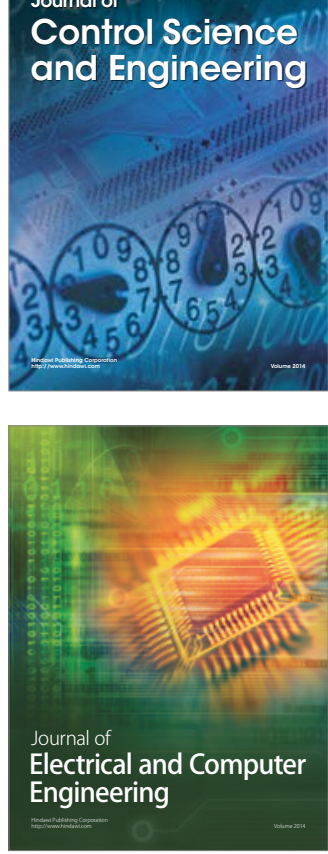

Distributed

Journal of

Control Science

and Engineering
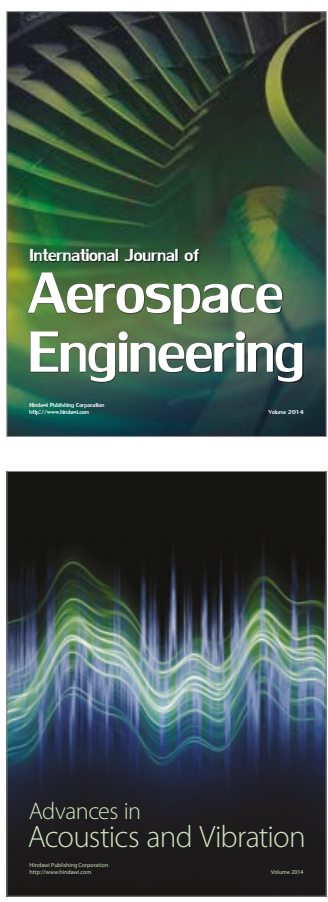

Sensor Networks 